\title{
Evaluation of Specimen Rejection rate in Hematology Laboratory.
}

\author{
Dr. Alpeshpuri P. Goswami *, Dr. Sher Sankar Roy **, \\ Dr. Nutanbala N. Goswami *** \\ *Associate Professor in Pathology, **Resident Doctor in Pathology, ***Associate Professor in \\ Pharmacology, Govt. Medical College, Bhavnagar-364001
}

\begin{abstract}
Objective: To calculate the rate of rejected specimens received in hematology laboratory stratified by area of collection and reason of rejection.

Design: Retrospective study conducted at Sir T General Hospital and Govt. Medical College, Bhavnagar, for twelve months period; January 1, 2013 to December 31, 2013. Data were retrieved from the laboratory records. Results: The rate of specimen rejection was highest in the medical ward and clotted specimens were the commonest cause for rejection followed by wrong patent identification.

Conclusion: A constellation of factors, mainly related to phlebotomy technique and wrong patient identification are the reasons for rejection of specimens in the hematology laboratory. The phlebotomy technique may be wrong, there may be lesser efficiency of the staff in phlebotomy and inability of correct patient identification maybe reasons for this observation.

Keywords:

Hematology

Anticoagulant

Rejection

Phlebotomy
\end{abstract}

\section{Introduction}

Sir T General Hospital and Govt. Medical College, Bhavnagar, is a government hospital, licensed for 780 beds. Care of the patient is administered as services for outpatient and inpatients as tertiary level hospital. Laboratory services are administered on routine and emergency basis. The hematology laboratory is one of the sections of laboratory services of hospital. The laboratory offers both inpatient and outpatient testing.Blood is either collected from the various wards for indoor patient and there is a separate blood collection area for outpatients. The medical laboratory is working for 24 hours / 7 days. A properly collected blood specimen is essential for quality performance in the laboratory.

Hematology testing is performed on whole blood. ${ }^{(1)}$

The laboratory data from a submitted specimen is reliable only if the specimen is adequately collected, labeled and transported to the laboratory in a timely fashion.

In our laboratory manual, in order to accept a specimen, it must meet the following criteria:

1- Must be collected in the correct specimen container, LAVENDER TOP K2 EDTA anticoagulant vaccutte for CBC, Reticulocyte count, AEC

2- Must be legibly identified.

The following information must be legibly recorded on a label $\&$ affixed in an irreversible fashion to the specimen container:-

- Patient's full name

- Medical Registration number

- Date and time of specimen collection

- Specimen source

- Signature/ initials of doctor

As soon as a blood specimen is withdrawn from a patient, it is mixed with an anticoagulant to prevent coagulation. The anticoagulant used for routine hematological tests is K2EDTA

where coagulation is prevented by the binding of calcium in the specimen to sites on the large EDTA molecule, thereby preventing the participation of the calcium in the coagulation cascade. ${ }^{(2)}$ 


\section{Objectives:}

Hematology specimens brought to the laboratory may be rejected if conditions are present that would compromise the validity of the test results.

Criteria we follow for specimen rejection at hematology department are the following:-

1. Improperly filled forms

2. Improperly labeled samples

3. Inadequate quantity of sample

4. Clotted sample

5. Spill over of sample

6. Haemolysed sample

7. Sample received after $4 \mathrm{hrs}$ of collection

8. Inappropriate vacute

9. Excessive amount

\section{Material and Methods}

A retrospective study was conducted at the Central laboratory of Haematology section of Pathology Deparment of Sir T General Hospital and Govt. Medical College, Bhavnagar from January 1, 2013 to December 31,2013 . The data were retrieved from the laboratory records.

\section{Results}

The total numbers of hematology samples as well as the number of rejected samples were collected. The areas of collection as well as the reason of rejection were recorded and the results were as follows.

Table No. 1 shows that overall rejection rate ranges from $0.17 \%$ to $4.68 \%$. Highest rejections seen from Orthopaedic ward (4.68\%). Followed by Critical Care Unit (4.59\%), Surgical Ward(4.25\%), Baby Medical Ward(3.5\%), Skin(3.26\%), Eye(3.03\%) and Gynae \& Obs(3.02\%). Lowest rejection rate is observed in Labside collection $(0.17 \%)$.

Table 1: Rejected Specimens - Site of collection

\begin{tabular}{|c|c|c|c|c|c|c|c|c|c|c|c|c|c|c|c|c|}
\hline MONTH & REJEC & MEDIC & $\mathrm{BM}$ & SURG & $\mathrm{CCU}$ & ORTHO & $\mathrm{G} \& \mathrm{O}$ & ICCU & EYE & ENT & CAS & ID & LAB & SKIN & TBW & PSY \\
\hline JAN '13 & 163 & 37 & 38 & 27 & 6 & 12 & 11 & 8 & 1 & 0 & 0 & 6 & 5 & 6 & 4 & 2 \\
\hline FEB'13 & 128 & 40 & 19 & 32 & 7 & 6 & 8 & 1 & 1 & 0 & 1 & 0 & 9 & 2 & 2 & 0 \\
\hline MAR'13 & 140 & 45 & 26 & 19 & 2 & 8 & 8 & 12 & 0 & 0 & 1 & 4 & 5 & 1 & 4 & 5 \\
\hline APR'13 & 128 & 42 & 24 & 21 & 3 & 6 & 7 & 1 & 0 & 2 & 0 & 7 & 7 & 1 & 6 & 1 \\
\hline MAY'13 & 108 & 32 & 9 & 20 & 9 & 2 & 11 & 9 & 0 & 1 & 1 & 4 & 4 & 0 & 3 & 3 \\
\hline JUNE'13 & 114 & 28 & 19 & 25 & 4 & 12 & 9 & 5 & 0 & 0 & 1 & 1 & 6 & 2 & 2 & 0 \\
\hline JULY'13 & 116 & 38 & 19 & 20 & 2 & 12 & 11 & 3 & 0 & 0 & 1 & 5 & 0 & 0 & 3 & 2 \\
\hline AUG'13 & 126 & 32 & 22 & 26 & 2 & 5 & 16 & 2 & 1 & 0 & 0 & 3 & 3 & 5 & 5 & 4 \\
\hline SEP'13 & 102 & 26 & 25 & 17 & 2 & 11 & 11 & 1 & 0 & 1 & 1 & 2 & 1 & 2 & 2 & 0 \\
\hline OCT'13 & 89 & 28 & 14 & 21 & 1 & 7 & 5 & 2 & 0 & 1 & 0 & 6 & 2 & 0 & 1 & 1 \\
\hline NOV'13 & 101 & 38 & 15 & 13 & 4 & 7 & 9 & 2 & 0 & 1 & 0 & 5 & 1 & 2 & 2 & 2 \\
\hline DEC'13 & 85 & 24 & 9 & 18 & 4 & 9 & 8 & 4 & 0 & 1 & 1 & 2 & 0 & 2 & 0 & 3 \\
\hline TOTAL & 1400 & 410 & 239 & 259 & 46 & 97 & 114 & 50 & 3 & 7 & 7 & 45 & 43 & 23 & 34 & 23 \\
\hline Received & 70477 & 16869 & 6804 & 6090 & 1002 & 2073 & 3777 & 2001 & 99 & 399 & 252 & 1560 & 25681 & 705 & 2121 & 1044 \\
\hline Rejec $(\%)$ & 1.99 & 2.43 & 3.513 & 4.2529 & 4.59 & 4.68 & 3.02 & 2.5 & 3.03 & 1.75 & 2.78 & 2.88 & 0.17 & 3.26 & 1.6 & 2.2 \\
\hline
\end{tabular}


Table 2: Rejected specimens, Reason of rejection

\begin{tabular}{|c|c|c|c|c|c|c|c|c|c|c|c|c|}
\hline Criteria & Jan & $\mathrm{Feb}$ & March & April & May & June & July & Aug & Sep & Oct & Nov & Dec \\
\hline Improperly filled forms $(2 \%)$ & 1 & 2 & 3 & 1 & 2 & 6 & 4 & 0 & 5 & 4 & 0 & 0 \\
\hline Improperly labeled samples ( $1.9 \%)$ & 2 & 2 & 1 & 1 & 1 & 2 & 7 & 0 & 1 & 9 & 0 & 1 \\
\hline Inadequate quantity of sample (3.86\%) & 3 & 6 & 5 & 5 & 3 & 7 & 4 & 14 & 3 & 4 & 0 & 0 \\
\hline Clotted sample $(78.57 \%)$ & 148 & 104 & 120 & 94 & 75 & 73 & 71 & 92 & 90 & 68 & 100 & 65 \\
\hline Spill over of sample (3.07\%) & 1 & 1 & 0 & 8 & & 10 & 8 & 7 & 1 & 2 & 2 & 3 \\
\hline Hemolysed Sample (7.64\%) & 3 & 5 & 7 & 13 & 19 & 12 & 14 & 4 & 1 & 0 & 3 & 16 \\
\hline Sample received after $4 \mathrm{hrs}$ of collection $(0 \%)$ & 0 & 0 & 0 & 0 & 0 & 0 & 0 & 0 & 0 & 0 & 0 & 0 \\
\hline Inappropriate vacute $(0.07 \%)$ & 0 & 1 & 0 & 0 & 0 & 0 & 0 & 0 & 0 & 0 & 0 & 0 \\
\hline Excessive $(3.57 \%)$ & 0 & 0 & 1 & 6 & 8 & 10 & 12 & 9 & 1 & 3 & 0 & 0 \\
\hline Total & 158 & 121 & 137 & 128 & 108 & 120 & 120 & 126 & 102 & 90 & 105 & 85 \\
\hline Grand Total & 1400 & & & & & & & & & & & \\
\hline
\end{tabular}

From table 2 it is seen that highest cause of rejection was clotted sample (78.57\%). Other causes of rejection observed were haemolysed sample (7.64\%), Inadequate quantity (3.86\%), Excessive quantity $(3.57 \%)$, Spill over of sample(3.07\%),Inappropriately filled forms(2\%), Inappropriately labeled sample(1.9\%) and lastly Inappropriate vacute $(0.07)$ respectively.

\section{Discussion}

Chart 1 : Rejected Specimens, Site of collection

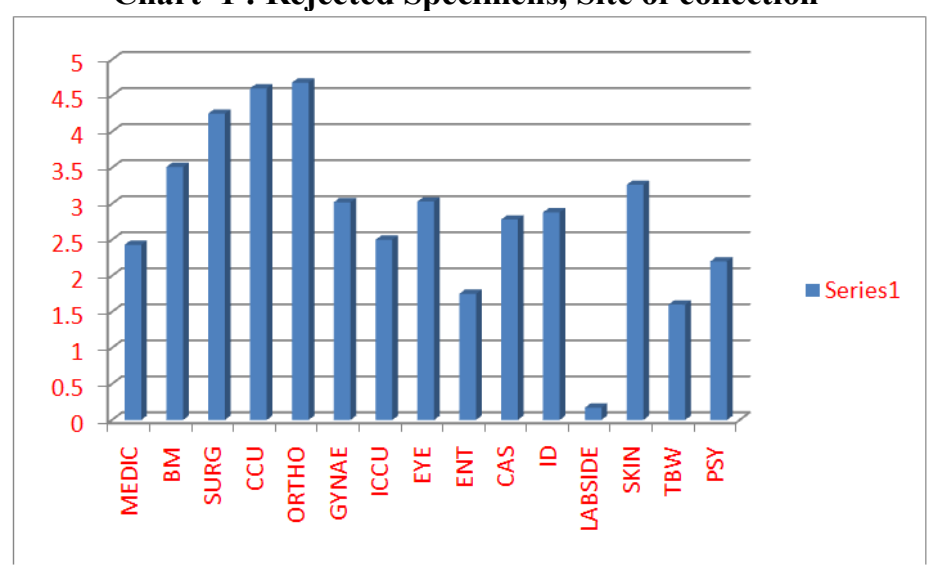

The highest rate of rejection was from the Orthopaedic ward, Critical care and Surgical ward, it was less in medical ward. High rejection rate in sensitive ward like Critical care unit and Baby medical ward is worrisome for hospital. As this causes unnecessary wastage of valuable time which may be life-saving for those seriously ill patients as well as repeated sample drawing is cumbersome and deteriorates the already compromised situation of patients. Again Surgical and surgical oriented wards are having higher rejection rate than medical and medical oriented wards. At Labside collection center the rejection rate is minimal especially in respect to the largest no. of specimen they receive. This proves that proper training, guidance and supervised collection can help to attain efficacy as well as minimize these preventable errors which are beneficial to patients and physicians. The most common cause for rejection was clotted specimen $(78.57 \%)$, followed by Haemolysed sample $(6.93 \%)$. These are due to mainly unnecessary delay between collection, lack of proper mixing of sample and delay in submission of sample. Rejected samples because of inappropriate or low quantity $(3.86 \%)$, excessive quantity $(3.57 \%)$ or spill over of sample $(3.07 \%)$ are mostly due to faulty technique by the staff collecting blood. Rejection due to improperly filled forms $(2 \%)$, improperly labeled samples $(1.9 \%)$ and inappropriate vacute $(0.07 \%)$ are mostly due to carelessness or to get the job done hurriedly. 


\section{Chart 2 : Rejected specimens, Reason of rejection}

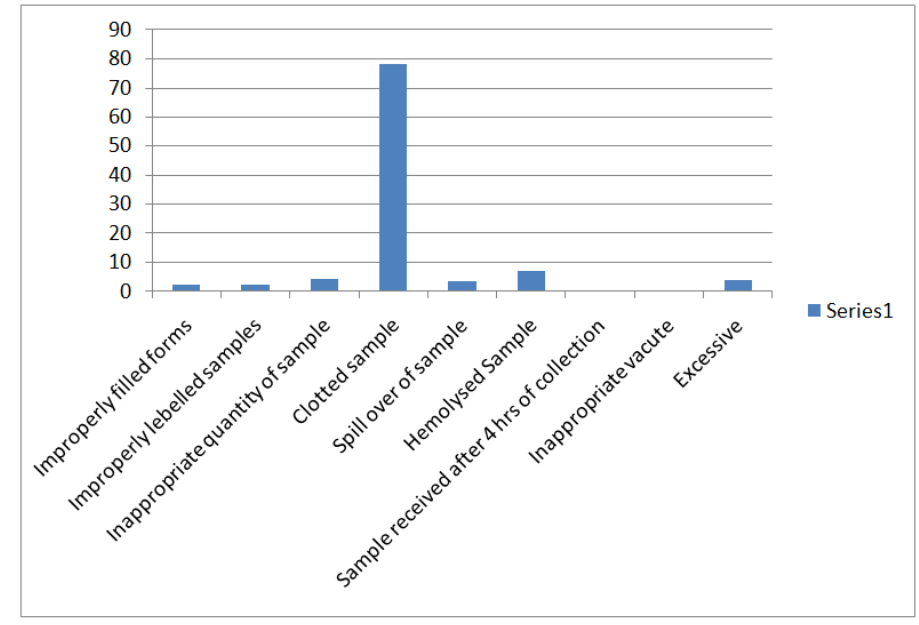

VI. Review of literature

The role of clinical pathology and laboratory medicine continues to grow as the single largest component in day to day clinical practice as well as long term follow-up. The result of any laboratory examination becomes good enough only if appropriate samples received in the laboratory.

Many national and international programs to track laboratory quality have reported laboratory specimen rejection rates ranging from $0.3 \%$ in outpatient facilities to $0.83 \%$ in hospital based laboratories. ${ }^{(4)}$

It is known that approximately $56 \%$ of laboratory error occurs during the preanalytic phase (processes that occur before testing of the sample) of laboratory testing. In 1997 Jones et al reported that preanalytical errors constituted between $25 \%$ and $50 \%$ of the total errors in the clinical laboratory. ${ }^{(5)}$

Preanalytical errors are the responsibilities of the blood collector and include the following: ${ }^{(2)}$

- Monitoring of specimen ordering

- Correct patient identification

- Patient communication and safety

- Patient preparation

- Timing of collections

- Phlebotomy equipment

- Collection techniques

- $\quad$ Specimen labeling

- Specimen transportation to the laboratory

- Specimen processing

In this study, we chose the hematology laboratory for calculating the rate of specimen rejection stratified by the area of collection and the reason for rejection. The results point clearly to a defect in the phlebotomy technique in addition to wrong patient identification, which should be targeted throughout the hospital with special emphasis on the orthopedic and surgical ward, since the highest rate of specimen rejection was encountered from there.

Recommendations to solve such issue is to organize a team of phlebotomists as well as laboratory staffs in conjunction with the quality department with the target of creating a procedure manual to provide health care personnel with concise information on the proper techniques to collect quality blood specimens with minimal patient discomfort. So as to minimize the errors encountered in blood collection that can lead to unsuitable specimens eventually rejected when received in the medical laboratory.

\section{References}

[1]. Turgeon, Mary Louise, "Clinical Hematology, Theory and Procedures." Boston/Toronto: Little, Brown and Co., 1988.

[2]. Blood Collection, A SHORT COURSEE D I T I O N 2

[3]. Arch Pathol Lab Med. 2007 Apr; 131(4):588-92.

[4]. Dale JC, Novis DA. Outpatient phlebotomy success and reasons for specimen rejection: a Q-Probes study. Arch Pathol Lab Med. 2002; 126:416-419.

[5]. Jones BA, Calam RR, Howanitz PJ. Chemistry specimen acceptability, a College of American Pathologists Q-Probes study of 453 laboratories. Arch Pathol Lab Med. 1997; 121:19-26. 